\title{
Differential Roles of Glial and Neuronal Glutamate Transporters in Purkinje Cell Synapses
}

\author{
Yukihiro Takayasu, ${ }^{1,2}$ Masae Iino, ${ }^{1}$ Wataru Kakegawa, ${ }^{1}$ Hiroshi Maeno, ${ }^{3}$ Kei Watase, ${ }^{3}$ Keiji Wada, ${ }^{3}$ Dai Yanagihara, ${ }^{4}$ \\ Taisuke Miyazaki, ${ }^{5}$ Okiru Komine, ${ }^{6}$ Masahiko Watanabe, ${ }^{5}$ Kohichi Tanaka, ${ }^{6}$ and Seiji Ozawa ${ }^{1}$ \\ Departments of ${ }^{1}$ Neurophysiology and ${ }^{2}$ Otolaryngology-Head and Neck Surgery, Gunma University Graduate School of Medicine, Maebashi, Gunma 371- \\ 8511, Japan, ${ }^{3}$ Department of Degenerative Neurological Diseases, National Institute of Neuroscience, Kodaira, Tokyo 187-8502, Japan, ${ }^{4}$ Laboratory for \\ Neurophysiology, Toyohashi University of Technology, Toyohashi, Aichi 441-8580, Japan, ${ }^{5}$ Department of Anatomy, Hokkaido University School of \\ Medicine, Sapporo 060-8638, Japan, and ' Laboratory of Molecular Neuroscience, School of Biomedical Science and Medical Research Institute, Tokyo \\ Medical and Dental University, Tokyo 113-8510, Japan
}

Glutamate transporters are essential for terminating excitatory neurotransmission. Two distinct glutamate transporters, glutamateaspartate transporter (GLAST) and excitatory amino acid transporter 4 (EAAT4), are expressed most abundantly in the molecular layer of the cerebellar cortex. GLAST is expressed in Bergmann glial processes surrounding excitatory synapses on Purkinje cell dendritic spines, whereas EAAT4 is concentrated on the extrasynaptic regions of Purkinje cell spine membranes. To clarify the functional significance of the coexistence of these transporters, we analyzed the kinetics of EPSCs in Purkinje cells of mice lacking either GLAST or EAAT4. There was no difference in the amplitude or the kinetics of the rising and initial decay phase of EPSCs evoked by stimulations of climbing fibers and parallel fibers between wild-type and EAAT4-deficient mice. However, long-lasting tail currents of the EPSCs appeared age dependently in most of Purkinje cells in EAAT4-deficient mice. These tail currents were never seen in mice lacking GLAST. In the GLAST-deficient mice, however, the application of cyclothiazide that reduces desensitization of AMPA receptors increased the peak amplitude of the EPSC and prolonged its decay more markedly than in both wild-type and EAAT4-deficient mice. The results indicate that these transporters play differential roles in the removal of synaptically released glutamate. GLAST contributes mainly to uptake of glutamate that floods out of the synaptic cleft at early times after transmitter release. In contrast, the main role of EAAT4 is to remove low concentrations of glutamate that escape from the uptake by glial transporters at late times and thus prevents the transmitter from spilling over to neighboring synapses.

Key words: glutamate transporters; GLAST; EAAT4; Purkinje cell; Bergmann glia; EPSC

\section{Introduction}

Glutamate transporters are essential for terminating excitatory neurotransmission. To date, five structurally related glutamate transporters have been cloned: excitatory amino acid transporter 1 (EAAT1)/glutamate-aspartate transporter (GLAST), EAAT2/ glutamate transporter 1 (GLT-1), EAAT3/excitatory amino acid carrier 1 (EAAC1), EAAT4, and EAAT5, in which GLAST and EAAT4 are expressed most abundantly in the molecular layer of the cerebellar cortex (Danbolt, 2001). GLAST is expressed in Bergmann glial processes surrounding excitatory synapses on Purkinje cells (PCs) (Chaudhry et al., 1995). EAAT4 is present in PC membranes, and the highest levels are found outside of the synapses on the dendritic spines (Tanaka et al., 1997). However, the

Received March 16, 2005; revised Aug. 9, 2005; accepted Aug. 9, 2005.

This work was supported by Grant-in-Aid 14208096 for Scientific Research (A) from the Japan Society for the Promotion of Sciences and Grant-in-Aid 15082202 for Scientific Research on Priority Areas on "Elucidation of glianeuron network mediated information processing systems" from the Ministry of Education, Culture, Sports, Science and Technology, Japan to S.0.

Correspondence should be addressed to Dr. Seiji Ozawa, Department of Neurophysiology, Gunma University Graduate School of Medicine, 3-39-22 Showa-machi, Maebashi, Gunma, 371-8511, Japan. E-mail: ozawas@med.gunma-u.ac.jp.

DOI:10.1523/JNEUROSCI.1020-05.2005

Copyright $\odot 2005$ Society for Neuroscience $\quad$ 0270-6474/05/258788-06\$15.00/0 functional significance of the coexistence of these glial and neuronal transporters around PC synapses is primarily unknown. To determine the contribution of GLAST and EAAT4 to the removal of synaptically released glutamate, we analyzed the kinetics of EPSCs elicited by stimulation of climbing fibers (CFs) and parallel fibers (PFs) in PCs in cerebellar slices prepared from GLAST- and EAAT4deficient mice (Watase et al., 1998; Huang et al., 2004).

\section{Materials and Methods}

Generation of mice deficient in transporters. EAAT4-/- mice and GLAST $-/$ - mice were generated as described previously (Watase et al., 1998; Huang et al., 2004). All mice had been inbred onto a C57BL/6 background for more than six generations.

Electrophysiological recordings. Parasagittal cerebellar slices (200-250 $\mu \mathrm{m})$ were prepared by using a microslicer. The solution used for slice storage and recording consisted of the following (in $\mathrm{mM}$ ): $125 \mathrm{NaCl}, 2.5$ $\mathrm{KCl}, 2 \mathrm{CaCl}_{2}, 1 \mathrm{MgCl}_{2}, 1.25 \mathrm{NaH}_{2} \mathrm{PO}_{4}, 26 \mathrm{NaHCO}_{3}$, and 10 D-glucose, saturated with $95 \% \mathrm{O}_{2}$ and $5 \% \mathrm{CO}_{2}$. Whole-cell patch-clamp recordings were performed from visualized PCs or Bergmann glia under a Zeiss (Oberkochen, Germany) Axioskop upright microscope at $32^{\circ} \mathrm{C}$. To block $\mathrm{GABA}_{\mathrm{A}}$ receptors, $100 \mu \mathrm{M}$ picrotoxin was added to the bathing solution. The pipette solution for EPSC recordings contained the following (in 
A CF-EPSCs

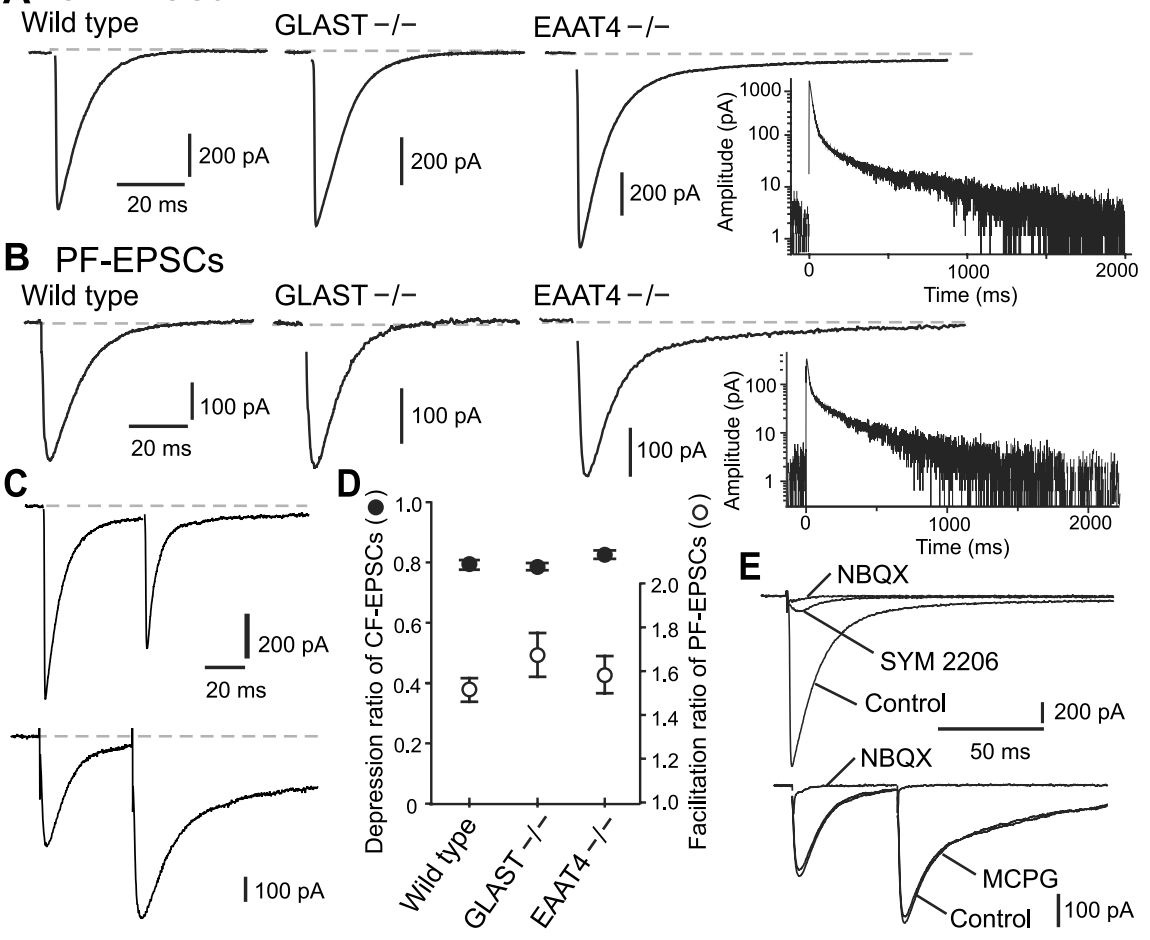

Figure 1. CF-EPSCS and PF-EPSCS in wild-type, GLAST-deficient, and EAAT4-deficient mice. $\boldsymbol{A}, \boldsymbol{B}$, Representative traces of CF-EPSCS $(\boldsymbol{A})$ and PF-EPSCS $(\boldsymbol{B})$. The insets show EPSCs in EAAT4-deficient mice on a log scale. $\boldsymbol{C}$, CF-EPSCs (top) and PF-EPSCS (bottom) in EAAT4-deficient mice evoked by a paired pulse. D, Degrees of paired-pulse depression of CF-EPSCs (filled circles) and facilitation of PF-EPSCS (open circles). The values for CF-EPSCS and PF-EPSCs were not significantly different among 11 and 10 cells in wild-type mice, 9 and 12 cells in GLAST-deficient mice, and 12 and 12 cells in EAAT4-deficient mice, respectively. Error bars represent SEM. E, CF-EPSCs in EAAT4-deficient mice in the absence and presence of $100 \mu \mathrm{m}$ SYM 2206 or $20 \mu \mathrm{m} \mathrm{NBQX} \mathrm{(top)} \mathrm{and}$ PF-EPSCs in EAAT4-deficient mice in the absence and presence of $1 \mathrm{~mm} \mathrm{MCPG}$ or $20 \mu \mathrm{M}$ NBQX (bottom). Note that a small component mediated by kainate receptor remained in the CF-EPSC trace in the presence of SYM 2206 (Huang et al., 2004).

changes in the expression of other glutamate transporter subtypes nor anatomical abnormalities (Watase et al., 1998; Huang et al., 2004) (supplementary Figs. S1 and S2, available at www.jneurosci.org as supplemental material). They also showed no changes in membrane properties of Bergmann glia (supplementary Table 1, available at www.jneurosci.org as supplemental material). Throughout this study, we evoked the PF-EPSCs by applying current pulses of $150-300 \mu \mathrm{A}$ in intensity so as to make its peak amplitude $\sim 350 \mathrm{pA}$, unless otherwise stated. There was no significant difference in the kinetics of the rising and initial decay phase of CF-EPSCs and PF-EPSCs among these mice (Fig. 1A,B). However, CF-EPSCs in EAAT4-deficient mice were followed by tail currents that lasted for nearly $2 \mathrm{~s}$ in 35 of 51 cells tested (69\%) (Fig. 1A). The mean amplitude measured at $50 \mathrm{~ms}$ after the onset of the EPSC was $6.8 \pm 0.6 \%$ of the peak amplitude $(n=35)$ in CF-EPSCs in EAAT4deficient mice. The CF synaptic currents in WT and GLAST-deficient mice returned completely to the baseline at this time point (Fig. 1A). The tail current was also detected in PF-EPSCs in all cells tested in EAAT4deficient mice (the mean tail current ratio, $5.2 \pm 0.7 \%$; $n=19$ ) (Fig. $1 B$ ). The pairedpulse stimulus given to CFs and PFs with a $50 \mathrm{~ms}$ interval induced paired-pulse depression and facilitation in the CF-EPSC and PF-EPSC, respectively (Fig. 1C). Their mean ratios showed no significant differences among WT, GLAST-, and EAAT4-

mm): 150 Cs-gluconate, $8 \mathrm{NaCl}, 2 \mathrm{MgATP}, 10$ HEPES, and $5 \mathrm{~N}$-ethyl bromide quaternary salt, $\mathrm{pH}$ 7.2. Synaptically activated transporter currents (STCs) in PCs and membrane properties of Bergmann glia were recorded with K-based pipette solution. Patch pipettes had a resistance of 2-4 M $\Omega$ when filled with the pipette solution. To evoke CF-EPSCs and PF-EPSCs in PCs, square pulses $(0.1 \mathrm{~ms}, 25-400 \mu \mathrm{A})$ were applied through a tungsten concentric bipolar electrode placed on the granule cell layer and the molecular layer, respectively. Recordings of EPSCs and STCs were taken at a holding potential of -10 and $-80 \mathrm{mV}$, respectively. The signals were filtered at $1-3 \mathrm{kHz}$ and digitized at $2-10 \mathrm{kHz}$. The current responses were recorded with an EPC-8 amplifier (HEKA, Darmstadt, Germany), and the pCLAMP system (Molecular Devices, Foster City, CA) was used for data acquisition and analysis. Data are expressed as means \pm SEM. The tail current ratio was defined as the relative amplitude of the tail current measured at $50 \mathrm{~ms}$ after the onset of the EPSC normalized to the peak amplitude, as shown in Figure $2 B$ (inset). The weighted decay is defined as the integrated EPSC after the peak divided by the peak amplitude. Statistical comparisons were performed by Student's $t$ test or one-way ANOVA with Scheffe's test for post hoc comparison. Differences were considered significant at $p<0.05$.

\section{Results}

\section{EPSCs in PCs in mice deficient in GLAST and EAAT4}

To investigate the functional roles of GLAST and EAAT4, we compared the properties of CF- and PF-mediated EPSCs among wild-type (WT), GLAST-, and EAAT4-deficient mice at postnatal day 22 (P22) to P42 (Fig. 1). Gene-deficient mice showed neither deficient mice, suggesting that the gene deletion caused no substantial change in release probability of PF or CF (Fig. $1 D)$. The tail currents of CF-EPSC and PF-EPSC were almost completely suppressed by $20 \mu \mathrm{M}$ 2,3-dioxo-6-nitro-1,2,3,4-tetrahydrobenzo[f] quinoxaline-7-sulfonamide (NBQX) or $100 \mu \mathrm{M}$ SYM 2206 (Li et al., 1999), a selective antagonist of AMPA receptors (Fig. $1 E$, top). However, $1 \mathrm{~mm}(R S)$ - $\alpha$-methyl-3-carboxymethylphenylglycine (MCPG), an antagonist of group I metabotropic glutamate receptors, had no effect on PF-EPSCs (Fig. $1 E$, bottom). Thus, the tail current was attributed predominantly to the activation of AMPA receptors.

The amplitude of the tail current to the second stimulus in the PF-EPSC was much greater than that to the first stimulus in EAAT4-deficient mice (Fig. 1C). The tail current ratios of PFEPSCs in EAAT4-deficient mice were $4.7 \pm 0.9$ and $31.7 \pm 3.1 \%$ $(n=15)$ of the respective peak amplitudes $(349 \pm 29$ and $557 \pm$ $45 \mathrm{pA}$ ) for the first and second pulses, respectively. The amplitude of the tail current in the PF-EPSC was dependent on the stimulus intensity. In Figure 2 A, PF-EPSCs were evoked in PCs in WT and EAAT4-deficient mice, respectively, by paired-pulse stimuli with a $50 \mathrm{~ms}$ interval and current intensity that varied from 70 to $370 \mu \mathrm{A}$. The tail current ratio in a representative cell is plotted in Figure $2 \mathrm{~B}$. These values to both the first and the second stimuli increased gradually with an increase in the current intensity. At the highest stimulus intensity, the tail current ratio 


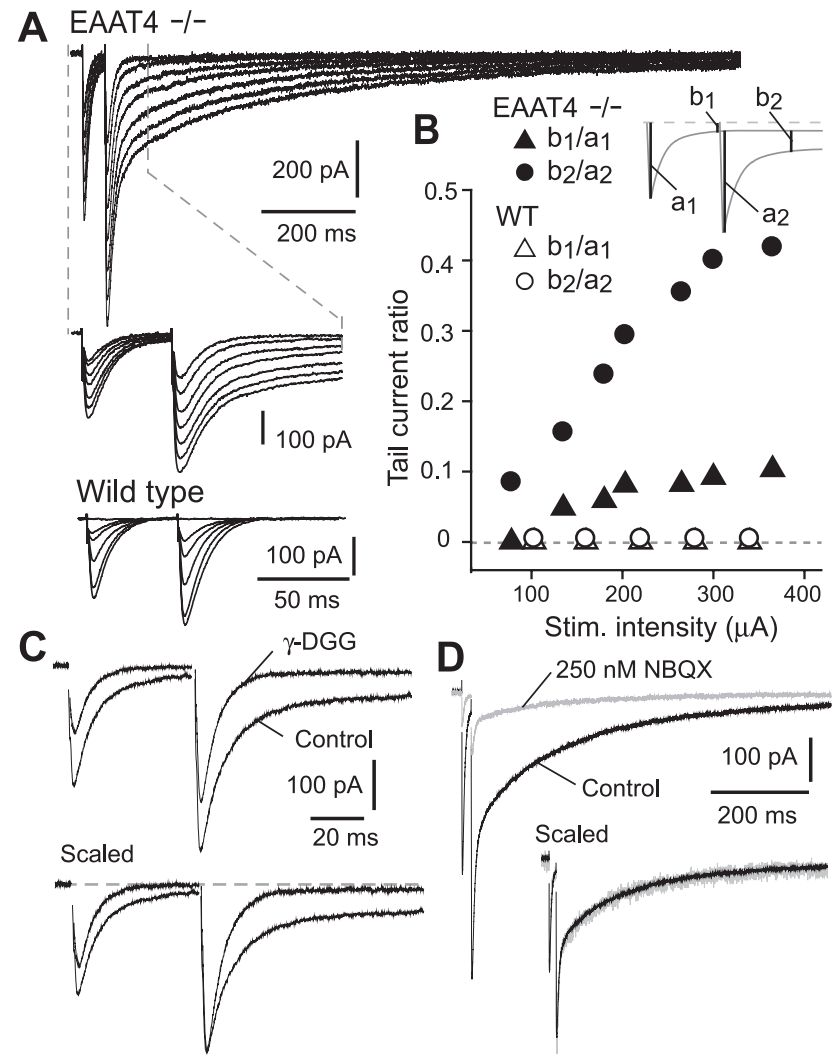

Figure 2. Dependence of the amplitude of tail current of the PF-EPSC on stimulus intensity in EAAT4-deficient mice and effects of antagonists of glutamate receptors. A, PF-EPSCs evoked by paired-pulse stimulations of various intensities in EAAT4-deficient (top and middle) and wildtype (bottom) mice. The middle traces show the initial part of the top traces on a fast time scale. $B$, Plots of tail current ratios of the EPSCs shown in $A$. The tail current ratios $\left(b_{1} / a_{1}\right.$ and $\left.b_{2} / a_{2}\right)$ for the first and the second stimuli with a $50 \mathrm{~ms}$ interval were defined as shown in the inset. Stim. Stimulus. C, D, Effects of $1 \mathrm{~mm} \gamma$-DGG $(\boldsymbol{C})$ and $250 \mathrm{~nm}$ NBQX (D) on PF-EPSCs. The bottom traces show EPSCs scaled to their peaks to the second stimuli.

reached $41.7 \pm 6.7 \%(n=6)$ in EAAT4-deficient mice. The corresponding values in WT mice were zero $(n=7)$.

EAAT4 is highly enriched in the extrasynaptic region of the PC dendritic spines (Tanaka et al., 1997). It is therefore likely that a portion of released glutamate would spill over to neighboring synapses (Carter and Regehr, 2000; Takayasu et al., 2004) and prolong the activation of glutamate receptors in EAAT4deficient mice. Actually, a low-affinity competitive antagonist of non-NMDA receptors, $\gamma$-D-glutamyl-glycine ( $\gamma$-DGG; 1 mM) (Watkins, 1991; Wadiche and Jahr, 2001), speeded the decay of PF-EPSC and abolished the tail current at $50 \mathrm{~ms}$ after the onset of the EPSC almost completely, whereas it reduced the peak amplitude induced by the second pulse of the paired stimulus by only $30.5 \pm 4.0 \%(n=7)$ in EAAT4 mice (Fig. $2 C)$. In contrast, a low concentration (250 nM) of NBQX reduced the amplitudes of the peak and tail currents equally by $\sim 90 \%$ and caused no change in the kinetics of both the EPSCs (Fig. 2D). $\gamma$-DGG (1 mM) also abolished the tail current of CF-EPSC almost completely but reduced its peak amplitude by only $14.4 \pm 0.6 \%(n=6$; data not shown). This preferential block of the tail current by $\gamma$-DGG strongly suggested that the tail current resulted from the activation of AMPA receptors by lower concentrations of glutamate, with which the low-

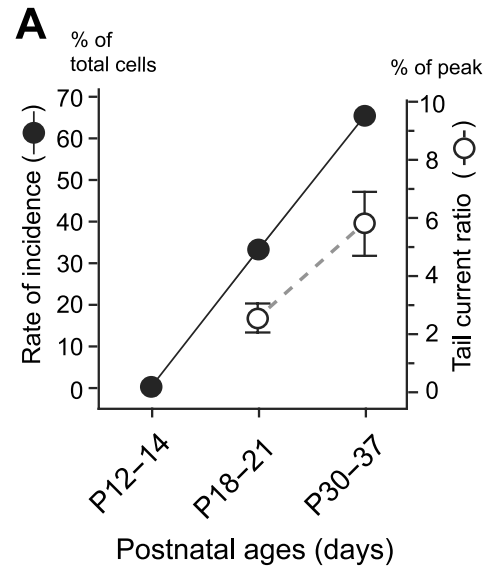

$\mathbf{B}_{\mathrm{P} 13}$

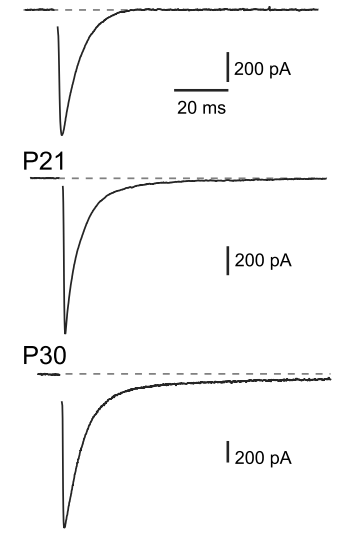

Figure 3. Age-dependent appearance of the tail current at $\mathrm{CF}$ synapses in EAAT4-deficient mice. $A$, Comparison of the incidence of the tail current in the three age groups and the mean of the tail current ratio of CF-EPSCS. The incidence represents the number of Purkinje cells with tail currents as percentage of the total cells tested. Filled circles, 0 of 24 at P12-P14, 7 of 22 at $P 18-P 21$, and 17 of 26 at P30 -P37. The mean of the tail current ratio was obtained from cells with tail currents for the two age groups. Open circles, $n=7$ at P18-P21 and $n=17$ at P30-P37. Error bars represent SEM. B, Representative traces of CF-EPSCs in EAAT4-deficient mice in the three age groups.

affinity competitive antagonist competed more effectively than it did with the higher concentrations of glutamate present at the peak of the EPSC (Wadiche and Jahr, 2001). These properties of the tail currents are well consistent with those of the slow-decayed EPSCs activated by glutamate spillover from neighboring synapses (Carter and Regehr, 2000; Takayasu et al., 2004). Furthermore, the notion that the loss of EAAT4 function causes the tail current in the EPSCs was supported by the finding that the bath application of a high concentration of TBOA (DL-threo- $\beta$-benzyloxyaspartate) (200 $\mu \mathrm{M}$ ), which suppresses all glutamate transporters nonselectively (Shimamoto et al., 1998), induced large tail currents similarly in WT and mutant mice (supplementary Fig. S3, available at www.jneurosci.org as supplemental material).

\section{Developmental changes in tail currents in EAAT4-deficient mice}

No tail current was shown in a previous report describing CF-EPSCs of P15-P19 EAAT4-deficient mice (Huang et al., 2004). In this study, we used P22-P42 mice. This suggested that the occurrence of the tail current in EAAT4-deficient mice was age dependent. To examine this possibility, we compared the incidence of the tail current and its amplitude in CF-EPSCs among three age groups: P12-P14, P18-P21, and P30-P37 (Fig. 3). No tail current was detected in P12-P14 mice $(n=24)$. A small tail current, the ratio of which was $2.5 \pm 0.2 \%$ of the peak amplitude, became detectable in 7 of 22 cells tested (32\%) in P18-P21 mice. The tail current was seen in 17 of 26 cells tested $(65 \%)$ in P30-P37 mice, and the mean of tail current ratio was $5.9 \pm 1.1 \%(n=17)$. We next examined the developmental changes in STCs in PCs in WT mice at the corresponding age groups (supplementary Fig. S4, available at www.jneurosci.org as supplemental material). The peak amplitudes of STCs were $9.5 \pm 1.3 \mathrm{pA}$ in P12-P14 mice $(n=5), 11.0 \pm 1.7 \mathrm{pA}$ in P18-P21 mice $(n=5)$, and $17.3 \pm 1.5 \mathrm{pA}$ in P30-P37 mice $(n=$ 


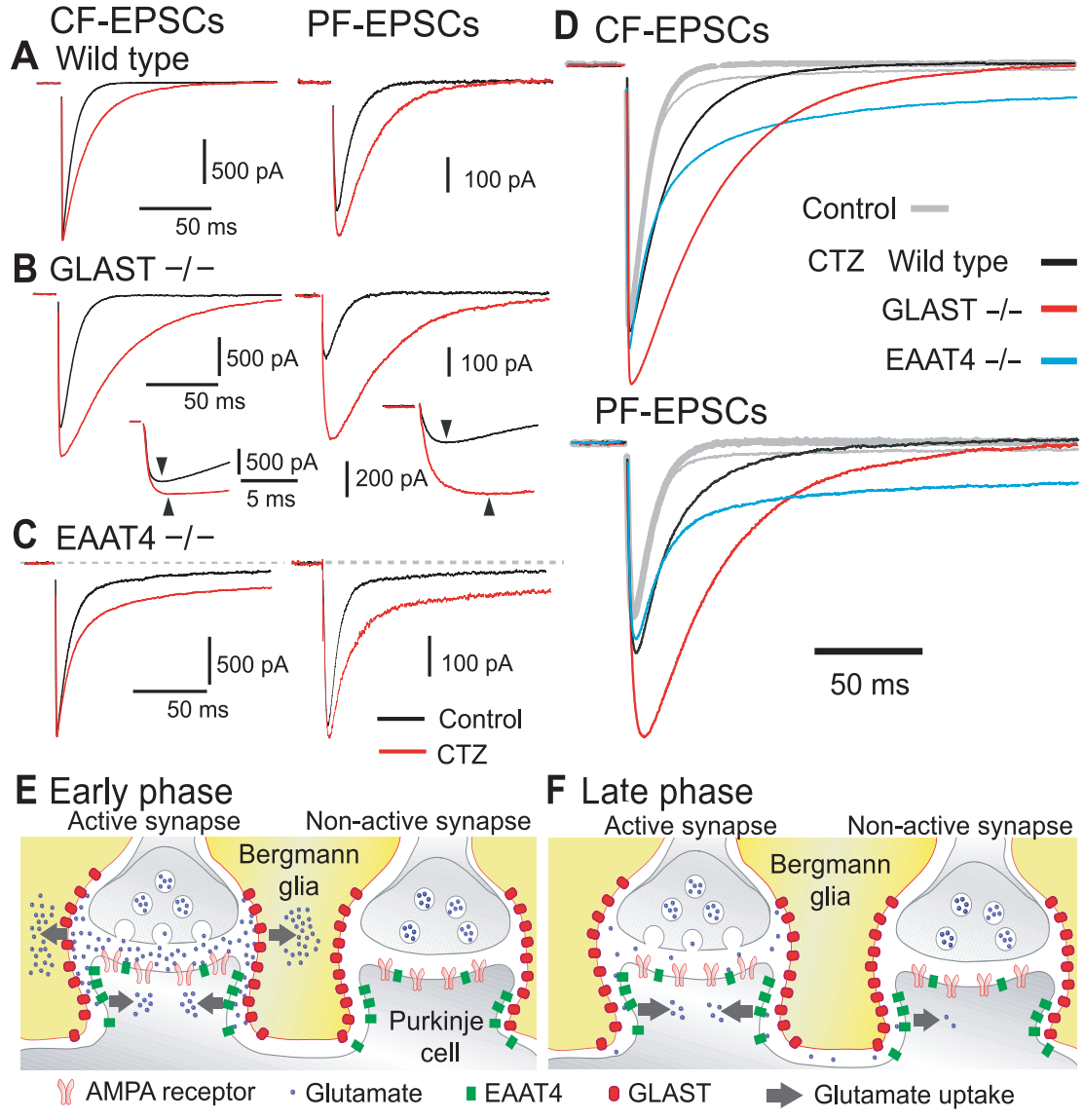

Figure 4. Comparison of EPSCs among wild-type, GLAST-deficient, and EAAT4-deficient mice in the presence of $100 \mu \mathrm{M}$ CTZ. $A-C$, EPSCs in the absence (black) and presence (red) of $100 \mu \mathrm{M}$ CTZ in wild-type ( $A)$, GLAST-deficient $(\boldsymbol{B})$, and EAAT4deficient $(\boldsymbol{C})$ mice. $\boldsymbol{D}$, Superimposition of the scaled EPSCs in control solution in wild-type, GLAST-deficient, and EAAT4deficient mice (gray) and the corresponding EPSC traces in the presence of $100 \mu \mathrm{M} \mathrm{CTZ} \mathrm{(black,} \mathrm{red,} \mathrm{and} \mathrm{blue} \mathrm{lines,} \mathrm{respec-}$ tively). The scaled traces in control solution are the averages of 13, 19, and 13 cells for CF-EPSCs and those of 11, 19, and 7 cells for PF-EPSCs, and the averages of the corresponding EPSC traces during the application of $100 \mu \mathrm{M}$ CTZ are further superimposed on them. In the presence of CTZ, the traces of CF-EPSC and PF-EPSC in GLAST-deficient mice crossed those in EAAT4deficient mice at 76 and $75 \mathrm{~ms}$ after the onset of the EPSC, respectively.E, $F$, Schematic diagrams illustrating differential roles of glial and neuronal glutamate transporters in Purkinje cell synapses. Synaptically released glutamate taken up by GLAST and EAAT4 in early $(\boldsymbol{E})$ and late $(\boldsymbol{F})$ phases of the synaptic event. Under physiological conditions, glutamate that escapes from the uptake by GLAST in the active synapse (left) is almost completely removed by EAAT4 and does not spill over to the nonactive synapse (right).

7). Thus, the activities of the neuronal glutamate transporter also increased age dependently.

\section{Comparison of kinetics of EPSCs between GLAST- and EAAT4-deficient mice}

There was no significant difference in the kinetics or the peak amplitude of either CF-EPSCs or PF-EPSCs between WT and GLAST-deficient mice in control solution under our experimental conditions (Watase et al., 1998). However, in the presence of $100 \mu \mathrm{M}$ cyclothiazide (CTZ), which reduces the desensitization of AMPA receptors and increases the affinity of glutamate to them (Partin et al., 1994), a clear difference was detected in the amplitude as well as kinetics of CF-EPSCs and PF-EPSCs between WT and GLAST-deficient mice (Fig. 4A,B). Although CTZ has been reported to increase the probability of transmitter release (Barnes-Davies and Forsythe, 1995), this effect is negligible, at least at CF synapses (Dzubay and Jahr, 1999). For CF-EPSCs, the application of $100 \mu \mathrm{M}$ CTZ significantly in- creased the $10-90 \%$ rise time from $0.41 \pm$ 0.02 to $0.51 \pm 0.03 \mathrm{~ms}(n=19 ; p<0.05)$ and the peak amplitude from $1021 \pm 59$ to $1262 \pm 72 \mathrm{pA}(n=19 ; p<0.05)$ in GLASTdeficient mice. This treatment caused no significant changes of these parameters of CF-EPSCs in WT mice, in agreement with the previous finding (Dzubay and Jahr, 1999). For PF-EPSC, the same treatment significantly increased the $10-90 \%$ rise time from $1.42 \pm 0.13$ to $2.04 \pm 0.18 \mathrm{~ms}$ $(n=11 ; p<0.05)$ and the peak amplitude from $343 \pm 38$ to $407 \pm 43 \mathrm{pA}(n=11 ; p<$ $0.05)$, even in WT mice. The effect of CTZ was more marked for PF-EPSCs in GLASTdeficient mice. The application of $100 \mu \mathrm{M}$ CTZ increased the $10-90 \%$ rise time from $1.42 \pm 0.03$ to $3.31 \pm 0.18 \mathrm{~ms}(n=19 ; p<$ $0.01)$ and the peak amplitude from $351 \pm$ 21 to $570 \pm 28 \mathrm{pA}(n=19 ; p<0.01)$ in GLAST-deficient mice. In contrast to the clear effect of CTZ on both rise time and peak amplitude of the EPSCs in GLASTdeficient mice, the effect of CTZ in EAAT4deficient mice was in a similar range to that in WT mice for both CF-EPSCs and PFEPSCs (Fig. 4C). However, we noted that $100 \mu \mathrm{M}$ CTZ markedly increased the amplitude of the tail currents of both the EPSCs in EAAT4-deficient mice (Fig. 4C). The application of $100 \mu \mathrm{M}$ CTZ increased the tail current ratios from $6.2 \pm 1.1$ to $26.1 \pm 4.5 \%$ of the peak amplitude for the CF-EPSC ( $n=$ 13 ) and $6.6 \pm 1.3$ to $30.1 \pm 3.8 \%$ of that for the $\operatorname{PF}-\operatorname{EPSC}(n=7)$.

To confirm the retardation of glutamate uptake in the early phase in GLASTdeficient mice, we compared the effects of $\gamma$-DGG on the peak amplitude and the decay of CF-EPSCs among WT, GLAST-, and EAAT4-deficient mice (supplementary Fig. S5, available at www.jneurosci.org as supplemental material). The peak amplitude of CF-EPSCs in WT, GLAST-, and EAAT4deficient mice were reduced by $27.0 \pm 4.0 \%$ $(n=6), 19.4 \pm 2.8 \%(n=6)$, and $29.3 \pm 2.4 \%(n=6)$ by $2 \mathrm{~mm}$ $\gamma$-DGG, respectively, and the degree of reduction was significantly smaller in GLAST-deficient mice than that in the other types of mice. Furthermore, in the presence of $2 \mathrm{~mm} \gamma$-DGG, the weighted decay of CF-EPSCs in GLAST-deficient mice $(7.0 \pm 0.3 \mathrm{~ms} ; n=6)$ was significantly slower than those in WT mice ( $5.8 \pm 0.1 \mathrm{~ms} ; n=6)$ and EAAT4-deficient mice $(6.0 \pm 0.2 \mathrm{~ms} ; n=6)$.

Finally, to compare the effects of the absence of GLAST and EAAT4 on the kinetics of the EPSCs, we superimposed the scaled CF-EPSCs or PF-EPSCs in control solution in WT, GLAST-, and EAAT4-deficient mice and further superimposed the corresponding EPSC traces in the presence of 100 $\mu \mathrm{M}$ CTZ on them in Figure $4 D$, in which each trace represents the average EPSCs of 7-19 PCs. The superimposed traces clearly revealed that the prolongation of the decay of the EPSC in the presence of CTZ was more marked in GLAST-deficient mice than in EAAT4-deficient mice in the early phase, whereas 
the opposite was true in the late phase (Fig. $4 D$ ). The traces in GLAST- and EAAT4-deficient mice crossed $\sim 75 \mathrm{~ms}$ after the onsets for both CF-EPSCs and PF-EPSCs. These differences in the kinetics of the prolonged EPSCs indicate that the retardation of the removal of synaptically released glutamate is more prominent in the early phase (up to $\sim 75 \mathrm{~ms}$ after the onset of the EPSC) in GLAST-deficient mice, whereas it is more prominent in the late phase (from $\sim 75 \mathrm{~ms}$ to $<2 \mathrm{~s}$ ) in EAAT4deficient mice in both CF and PF synapses.

\section{Discussion}

Our results indicate that GLAST works mainly at early times after transmitter release to remove high concentrations of glutamate that overflow to the nearest processes of Bergmann glia wrapping synaptic clefts (Fig. $4 E$ ), whereas EAAT4 is more responsible for removal of low concentrations of glutamate that remain in the extrasynaptic region at late times and effectively preventing glutamate from spilling over to neighboring synapses (Fig. $4 F$ ). The $\sim 10$-fold higher affinity of glutamate to EAAT4 than that to GLAST (Arriza et al., 1994; Fairman et al., 1995) enables these transporters to play their distinct roles in glutamatergic synapses of PCs. However, our data do not exclude the possibility that EAAT4 takes up a part of released glutamate at early times. Although EAAT4 is highly enriched in the extrasynaptic region of the PC dendritic spines, it is present at low concentrations in the synaptic membrane (Danbolt, 2001) (Fig. 4E,F). Furthermore, PCs were held at $-10 \mathrm{mV}$ using a $\mathrm{Cs}^{+}$-based pipette solution in this study. This recording condition would inhibit partly the activity of EAAT4, and therefore the contribution of EAAT4 to removal of glutamate at early times would be underestimated. It is also possible that GLAST partly works to remove glutamate at late times because it is expressed abundantly in the extrasynaptic region (Danbolt, 2001).

The relative contribution of glial and neuronal transporters to the uptake of glutamate at CF synapses has been estimated previously by analyzing both stoichiometric and anion currents associated with glutamate transport in PCs. Estimates based on anion currents suggested that $\sim 22 \%$ of glutamate released at CF synapses was removed by postsynaptic trasnsporters, presumably by EAAT4 (Otis et al., 1997). In contrast, measurements based on stoichiometric transporter currents suggested that most of the released glutamate (56-230\%) was removed by postsynaptic trasnsporters (Auger and Attwell, 2000). However, the latter has been shown to be an overestimation of the amount of glutamate removed by EAAT4, because the stoichiometric transporter current was contaminated by the kainate receptor-mediated current (Huang et al., 2004). Very recently, the contribution of EAAT4 to glutamate uptake has been estimated to be only $\sim 17 \%$ by taking the kainate receptor-mediated component of the synaptic current into consideration (Brasnjo and Otis, 2004). Thus, the majority of released glutamate is presumably taken up by Bergmann glial cells. This notion is consistent with our results that high concentrations of glutamate at early times after transmitter release are removed by GLAST.

The tail current was not detected in CF-EPSCs at P15-P19 in mice lacking in EAAT4 or both EAAT4 and EAAC1 (Huang et al., 2004). In this study, we found that the occurrence of the tail current was age dependent in EAAT4-deficient mice. This suggests that the chance for cross-talk by glutamate spillover between neighboring synapses increases when excitatory synapses on PC dendrites become more mature with animal de- velopment. Interestingly, expression of EAAT4 protein increases with maturation. EAAT4 is expressed at a very low level at $\mathrm{P} 10$ in rats and then substantially increases at P16, reaching a maximum level at P26 (Furuta et al., 1997). In the molecular layer of the mouse cerebellum, the immunoreactivity for EAAT4 markedly increases during the third week of life (Yamada et al., 1997). It is most likely that EAAT4 is needed to prevent glutamate from spilling over to neighboring synapses in mature PCs. The contribution of EAAC1 to removal of synaptically released glutamate may be almost negligible because its expression around PC synapses is extremely small (Furuta et al., 1997), and no EAAC1-mediated STC is detected (Huang et al., 2004).

An obvious question is which changes underlie the agedependent appearance of tail currents in EAAT4-deficient mice. The activation of AMPA receptors in neighboring synapses by glutamate spillover would become more marked when either the density of excitatory synapses in the PC or the density of AMPA receptors at each synapse would increase during development (Diamond, 2002). Alternatively, changes in the geometry of the extracellular space with glial sheaths as diffusion barriers would occur (Barbour et al., 1994; Lehre and Danbolt, 1998). If the dendritic spine became covered by glial processes more tightly during development, synaptic spillover retaining a high level of glutamate would cause cross-talk between neighboring synapses more easily.

\section{References}

Arriza JL, Fairman WA, Wadiche JI, Murdoch GH, Kavanaugh MP, Amara SG (1994) Functional comparison of three glutamate transporter subtypes cloned from human motor cortex. J Neurosci 14:5559-5569.

Auger C, Attwell D (2000) Fast removal of synaptic glutamate by postsynaptic transporters. Neuron 28:547-558.

Barbour B, Keller BU, Llano I, Marty A (1994) Prolonged presence of glutamate during excitatory synaptic transmission to cerebellar Purkinje cells. Neuron 12:1331-1343.

Barnes-Davies M, Forsythe ID (1995) Pre- and postsynaptic glutamate receptors at a giant excitatory synapse in rat auditory brainstem slices. J Physiol (Lond) 488:387-406.

Brasnjo G, Otis TS (2004) Isolation of glutamate transport-coupled charge flux and estimation of glutamate uptake at the climbing fiber-Purkinje cell synapse. Proc Natl Acad Sci USA 101:6273-6278.

Carter AG, Regehr WG (2000) Prolonged synaptic currents and glutamate spillover at the parallel fiber to stellate cell synapse. J Neurosci 20:4423-4434.

Chaudhry FA, Lehre KP, van Lookeren Campagne M, Ottersen OP, Danbolt NC, Storm-Mathisen J (1995) Glutamate transporters in glial plasma membranes: highly differentiated localizations revealed by quantitative ultrastructural immunocytochemistry. Neuron 15:711-720.

Danbolt NC (2001) Glutamate uptake. Prog Neurobiol 65:1-105.

Diamond JS (2002) A broad view of glutamate spillover. Nat Neurosci 5:291-292.

Dzubay JA, Jahr CE (1999) The concentration of synaptically released glutamate outside of the climbing fiber-Purkinje cell synaptic cleft. J Neurosci 19:5265-5274.

Fairman WA, Vandenberg RJ, Arriza JL, Kavanaugh MP, Amara SG (1995) An excitatory amino-acid transporter with properties of ligand-gated chloride channel. Nature 375:599-603.

Furuta A, Rothstein JD, Martin LJ (1997) Glutamate transporter protein subtypes are expressed differentially during rat CNS development. J Neurosci 17:8363-8375.

Huang YH, Dykes-Hoberg M, Tanaka K, Rothstein JD, Bergles DE (2004) Climbing fiber activation of EAAT4 transporters and kainate receptors in cerebellar Purkinje cells. J Neurosci 24:103-111.

Lehre KP, Danbolt NC (1998) The number of glutamate transporter subtype molecules at glutamatergic synapses: chemical and stereological quantification in young adult rat brain. J Neurosci 18:8751-8757. 
Li P, Wilding TJ, Kim SJ, Calejesan AA, Huettner JE, Zhuo M (1999) Kainate-receptor-mediated sensory synaptic transmission in mammalian spinal cord. Nature 397:161-164.

Otis TS, Kavanaugh MP, Jahr CE (1997) Postsynaptic glutamate transport at the climbing fiber-Purkinje cell synapse. Science 277:1515-1518.

Partin KM, Patneau DK, Mayer ML (1994) Cyclothiazide differentially modulates desensitization of $\alpha$-amino-3-hydroxy-5-methyl-4-isoxazolepropionic acid receptor splice variants. Mol Pharmacol 46:129-138.

Shimamoto K, LeBrun B, Yasuda-Kamatani Y, Sakatani M, Shigeri Y, Yumoto N, Nakajima T (1998) DL-threo- $\beta$-benzyloxyaspartate, a potent blocker for excitatory amino acid transporter. Mol Pharmacol 53:195-201.

Takayasu Y, Iino M, Ozawa S (2004) Roles of glutamate transporters in shaping excitatory synaptic currents in cerebellar Purkinje cells. Eur J Neurosci 19:1285-1295.
Tanaka J, Ichikawa R, Watanabe M, Tanaka K, Inoue Y (1997) Extrajunctional localization of glutamate transporter EAAT4 at excitatory Purkinje cell synapses. NeuroReport 8:2461-2464.

Wadiche JI, Jahr CE (2001) Multivesicular release at climbing fiberPurkinje cell synapses. Neuron 32:301-313.

Watase K, Hashimoto K, Kano M, Yamada K, Watanabe M, Inoue Y, Okuyama S, Sakagawa T, Ogawa S, Kawashima N, Hori S, Takimoto M, Wada K, Tanaka K (1998) Motor discoordination and increased susceptibility to cerebellar injury in GLAST mutant mice. Eur J Neurosci 10:976-988.

Watkins JC (1991) Some chemical highlights in the development of excitatory amino acid pharmacology. Can J Physiol Pharmacol 69:1064-1075.

Yamada K, Wada S, Watanabe M, Tanaka K, Wada K, Inoue Y (1997) Changes in expression and distribution of the glutamate transporter EAAT4 in developing mouse Purkinje cells. Neurosci Res 27:191-198. 